\title{
MATRIX SUMMABILITY OF CONVEX SEQUENCES
}

\author{
DAVID F. DAWSON
}

The classical Silverman-Toeplitz theorem states that a matrix $A=\left(a_{p q}\right)$ sums every convergent sequence if and only if

(1) $\left\{a_{p q}\right\}_{p=1}^{\infty}$ converges, $q=1,2,3, \cdots$,

(2) $\left\{\sum_{q=1}^{\infty} a_{p q}\right\}_{p=1}^{\infty}$ converges, and

(3) there exists a number $k$ such that $\sum_{q=1}^{\infty}\left|a_{p q}\right|<k, p=1,2,3, \cdots$. Hahn [2] showed that $A$ sums every sequence of bounded variation, i.e. every absolutely convergent sequence, if and only if (1), (2), and

$(3)^{\prime}$ there exists a number $k$ such that $\left|\sum_{q=1}^{n} a_{p q}\right|<k, p, n=1,2$, $3, \cdots$.

In this paper we obtain three conditions which are necessary and sufficient for a matrix to sum every convergent convex sequence. The first two conditions are (1) and (2) above, while the third condition is a weakened version of $(3)^{\prime}$. Matrices considered here have complex elements even though convex sequences are necessarily real sequences.

The following lemma embodies well-known properties of convex sequences which we will need.

Leмma. If $\left\{\mu_{p}\right\}$ is a bounded convex sequence, then

(1) $\left\{\mu_{p}\right\}$ is nonincreasing and convergent,

(2) $n \Delta \mu_{n} \rightarrow 0$ as $n \rightarrow \infty$, where $\Delta \mu_{n}=\mu_{n}-\mu_{n+1}$,

(3) $\sum_{p=1}^{\infty} p \Delta^{2} \mu_{p}=\mu_{1}-\lim \mu_{n}$, where $\Delta^{2} \mu_{p}=\Delta \mu_{p}-\Delta \mu_{p+1}$.

The lemma not only shows that the set of all convergent convex sequences is a subset of the set $S_{\mathrm{BV}}$ of all sequences of bounded variation, but it can be used in the following way to show that the finite linear completion (using complex coefficients) of the set of all convergent convex sequences is a proper subset of $S_{\mathrm{Bv}}$. It is trivial to construct a nonincreasing null sequence (hence a sequence in $S_{\mathrm{BV}}$ ) $\left\{\mu_{p}\right\}$ such that $\lim p \Delta \mu_{p} \neq 0$. Hence, according to the lemma, such a sequence cannot be a finite linear combination of convergent convex sequences. Therefore the conditions (1), (2), and (3)' of Hahn, while sufficient for a matrix to sum every sequence in $S_{\mathrm{Bv}}$, may not be necessary for a matrix to sum every convergent convex sequence, and indeed they are not necessary, as shown by our theorem which follows.

ThEOREM. In order for a matrix $A=\left(a_{p q}\right)$ to sum every convergent

Presented to the Society, January 23, 1968; received by the editors May 26, 1967. 
convex sequence, it is necessary and sufficient that the following conditions hold:

(i) $\left\{a_{p q}\right\}_{p=1}^{\infty}$ converges, $q=1,2,3, \cdots$,

(ii) $\left\{\sum_{q=1}^{\infty} a_{p q}\right\}_{p=1}^{\infty}$ converges, and

(iii) there exists a number $k$ such that

$$
\left|\sum_{j=1}^{n} \sum_{q=1}^{j} a_{p q}\right|<n k, \quad p, n=1,2,3, \cdots .
$$

Proof. We will show first that (i), (ii), and (iii) are sufficient. From (ii) we see that $A$ sums every constant term sequence. Hence we need only show that $A$ sums every convex sequence which converges to zero. Let $x=\left\{x_{p}\right\}$ be a convex null sequence. Then from (1) of the lemma, $x$ is a nonincreasing sequence. Let $j$ be a positive integer. Then from a theorem of Hadamard [1] we see that $\sum_{p=1}^{\infty} a_{j p} x_{p}$ is convergent since $\sum_{p=1}^{\infty} a_{j p}$ converges and $x$ is of bounded variation. Thus $A x$ is a sequence. If $n$ is a positive integer, then, using summation by parts, we have

$$
\begin{aligned}
\sum_{p=1}^{n} a_{j p} x_{p}= & a_{j 1} \Delta x_{1}+\left(a_{j 1}+a_{j 2}\right) \Delta x_{2} \\
& +\cdots+\left(\sum_{p=1}^{n-1} a_{j p}\right) \Delta x_{n-1}+\left(\sum_{p=1}^{n} a_{j p}\right) x_{n} .
\end{aligned}
$$

Let $t_{j p}=a_{j 1}+a_{j 2}+\cdots+a_{j p}, p=1,2,3, \cdots$. From (ii) there exists a number $k_{j}$ such that $\left|t_{j p}\right|<k_{j}, p=1,2,3, \cdots$. Hence from $\left(^{*}\right)$ we have $\sum_{p=1}^{\infty} a_{j p} x_{p}=\sum_{p=1}^{\infty} t_{j p} \Delta x_{p}$, since $\left|t_{j n} x_{n}\right| \leqq k_{j} x_{n} \rightarrow 0$ as $n \rightarrow \infty$. But

$$
\begin{aligned}
\sum_{p=1}^{n} t_{j p} \Delta x_{p}= & t_{j 1} \Delta^{2} x_{1}+\left(t_{j 1}+t_{j 2}\right) \Delta^{2} x_{2} \\
& +\cdots+\left(\sum_{p=1}^{n-1} t_{j p}\right) \Delta^{2} x_{n-1}+\left(\sum_{p=1}^{n} t_{j p}\right) \Delta x_{n} .
\end{aligned}
$$

Let $s_{j p}=t_{j 1}+t_{j 2}+\cdots+t_{j p}, p=1,2,3, \cdots$. Then from $\left(^{\circ}\right)$ we have $\sum_{p=1}^{\infty} t_{j p} \Delta x_{p}=\sum_{p=1}^{\infty} s_{j p} \Delta^{2} x_{p}$, since $\left|s_{j n} \Delta x_{n}\right| \leqq n k_{j} \Delta x_{n}$ and from (2) of the lemma $n \Delta x_{n} \rightarrow 0$ as $n \rightarrow \infty$. Thus $\sum_{p=1}^{\infty} a_{j p} x_{p}=\sum_{p=1}^{\infty} s_{j p} \Delta^{2} x_{p}$. Let $u_{j p}=s_{j p} / p, p=1,2,3, \cdots$, and let $U=\left(u_{p q}\right)$. Let $y=\left\{y_{p}\right\}=\left\{p \Delta^{2} x_{p}\right\}$. Then $A x=U y$. We wish to show now that $U y$ is convergent. From (iii) we have $\left|u_{p q}\right|<k, p, q=1,2,3, \cdots$. We note from (3) of the lemma that $\sum y_{p}=\sum p \Delta^{2} x_{p}$ is convergent. We note from (i) that $U$ has convergent columns. Let $u_{p}=\lim _{q} u_{q p}, p=1,2,3, \cdots$. Then $\left|u_{p}\right| \leqq k, p=1,2,3, \cdots$. Clearly $\sum u_{p} y_{p}$ is convergent. Let $\epsilon>0$. Let $M$ be a positive integer such that $k \sum_{p=M+1}^{\infty} y_{p}<\epsilon / 3$. Let $Q$ 
$=1+\sum_{p=1}^{M} y_{p}$. Let $N$ be a positive integer such that if $n>N$ and $1 \leqq p \leqq M$, then $\left|u_{n p}-u_{p}\right|<\epsilon /(3 Q)$. Then if $n>N$, we have

$$
\begin{aligned}
\left|\sum_{p=1}^{\infty} u_{n p} y_{p}-\sum_{p=1}^{\infty} u_{p} y_{p}\right| & \leqq \\
& +\left|\sum_{p=1}^{M}\left(u_{n p}-u_{p}\right) y_{p}\right|+\left|\sum_{p=M+1}^{\infty} u_{p} y_{p}\right|<\epsilon .
\end{aligned}
$$

Thus $U y$ is convergent, and so $A x$ is convergent. Hence the fact that (i), (ii), and (iii) are sufficient for $A$ to sum every convergent convex sequence has been established.

We now consider the converse. Clearly if $A$ sums every convergent convex sequence, then (ii) holds since $\{1,1,1, \ldots\}$ is such a sequence.

If $A$ sums every convergent convex sequence, then the first column of $A$ converges, since $\{1,0,0,0, \ldots\}$ is a convergent convex sequence. Thus the second column of $A$ is convergent, since $\{2,1,0$, $0,0, \ldots\}$ is a convergent convex sequence. Hence a simple induction argument can be used to show that $A$ has convergent columns, i.e. (i) holds.

Suppose $A$ sums every convergent convex sequence. We wish to show that (iii) holds. Since (ii) holds, there exists a number $R$ such that $\left|\sum_{q=1}^{\infty} a_{p q}\right|<R, p=1,2,3, \cdots$. Using the notation introduced in the proof of the sufficiency, we note that $\lim _{q} u_{p q}=\sum_{j=1}^{\infty} a_{p j}, p$ $=1,2,3, \ldots$. Assume that (iii) does not hold. There exists an element $u_{p_{1} q_{1}}$ of the matrix $U$ such that

$$
\left|u_{p_{1} q_{1}}\right|>4^{2}(1+R) \text {. }
$$

Let $N_{1}$ be a positive integer such that if $q>N_{1}$, then $\left|u_{p_{1} q}\right|<R$. There exists a positive integer $M_{1}$ such that if $p>M_{1}$, then $\left|u_{p q_{1}}-u_{q_{1}}\right|<1$. Let $Q_{1}=1+\left|u_{q_{1}}\right|$, and let $q_{0}=0$. Since $U$ has convergent columns and (iii) does not hold, there exists an element $u_{p_{2} q_{2}}$ of $U$ such that $p_{2}>M_{1}, q_{2}>N_{1}, q_{2}-q_{1}>q_{1}-q_{0}$, and

$$
\left|u_{p_{2} q_{2}}\right|>4^{3}\left(1+R+Q_{1}\right) \text {. }
$$

Let $N_{2}$ be a positive integer such that if $q>N_{2}$, then $\left|u_{p_{2} q}\right|<R$. There exists an integer $M_{2}>M_{1}$ such that if $p>M_{2}$, then $\left|u_{p q_{2}}-u_{q_{2}}\right|<1$. Let $Q_{2}=1+\left|u_{q_{2}}\right|$. There exists an element $u_{p_{3} q_{3}}$ of $U$ such that $p_{3}>M_{2}, q_{3}>N_{2}, q_{3}-q_{2}>q_{2}-q_{1}$, and

$$
\left|u_{p_{3} q_{3}}\right|>4^{4}\left(1+R+Q_{1}+Q_{2}\right) \text {. }
$$

Continue the process. 
If $n$ is a positive integer, let $L_{n}$ be the line which contains the points $\left(q_{n-1}+1,1 / 2^{n-1}\right)$ and $\left(q_{n}+1,1 / 2^{n}\right)$. We define a sequence $x=\left\{x_{p}\right\}$ as follows. If $n$ is a positive integer and $q_{n-1}+1 \leqq t<q_{n}+1$, then $x_{t}$ is the ordinate of the point on line $L_{n}$ with abscissa $t$. Let $m$ be a positive integer. Then

$$
\begin{aligned}
\text { slope of } L_{m} & =-\left(1 / 2^{m}\right) /\left(q_{m}-q_{m-1}\right), \\
\text { slope of } L_{m+1} & =-\left(1 / 2^{m+1}\right) /\left(q_{m+1}-q_{m}\right),
\end{aligned}
$$

and so

$$
\begin{aligned}
\Delta^{2} x_{q_{m}} & =\left(1 / 2^{m}\right) /\left(q_{m}-q_{m-1}\right)-\left(1 / 2^{m+1}\right) /\left(q_{m+1}-q_{m}\right) \\
& >\left(1 / 2^{m}\right) /\left(q_{m}-q_{m-1}\right)-\left(1 / 2^{m+1}\right) /\left(q_{m}-q_{m-1}\right) \\
& =1 / 2^{m+1}\left(q_{m}-q_{m-1}\right) .
\end{aligned}
$$

We note that $\Delta^{2} x_{p}=0$ if there is no $j$ such that $p=q_{j}$. Thus $x$ is a convex null sequence, and

$$
q_{p} \Delta^{2} x_{q_{p}}>1 / 2^{p+1}, \quad p=1,2,3, \cdots .
$$

Let $n$ be an integer greater than 1 . Then

$$
\begin{aligned}
\left|\sum_{j=1}^{\infty} u_{p_{n} j} \Delta^{2} x_{j}\right| \geqq & \left|u_{p_{n} q_{n}} q_{n} \Delta^{2} x_{q_{n}}\right|-\left|\sum_{j=1}^{n-1} u_{p_{n} q_{j}} q_{j} \Delta^{2} x_{q_{j}}\right|-\left|\sum_{j=n+1}^{\infty} u_{p_{n} q_{j}} q_{j} \Delta^{2} x_{q_{j}}\right| \\
> & \frac{4^{n+1}\left(1+R+Q_{1}+\cdots+Q_{n-1}\right)}{2^{n+1}} \\
& -\sum_{j=1}^{n-1} Q_{j}-R \sum_{j=n+1}^{\infty} q_{j} \Delta^{2} x_{q_{j}}>2^{n+1},
\end{aligned}
$$

since, from (3) of the lemma, $\sum_{j=1}^{\infty} j \Delta^{2} x_{j}=1$. Let $y=\left\{y_{p}\right\}=\left\{p \Delta^{2} x_{p}\right\}$. Thus $A x=U y$ is divergent. Hence the assumptions that $A$ sums every convergent convex sequence and that (iii) does not hold lead to a contradiction. Thus if $A$ sums every convergent convex sequence, then (i), (ii), and (iii) hold. This completes the proof of the theorem.

\section{REFERENCES}

1. J. Hadamard, Deux thêorèmes d'Abel sur la convergence des sêries, Acta Math. 27 (1903), 177-183. 3-88.

2. Hans Hahn, Über Folgen linearer Operationen, Monatsh. Math. 32 (1922),

North Texas State University 